\title{
QUANTITATIVE ANALYSIS OF GATED MYOCARDIAL PERFUSION SPECT
}

\author{
Victor Ploskikh \\ Saint Petersburg State University \\ Russia \\ v.ploskikh@spbu.ru
}

Article history:

Received 03.12.2018, Accepted 19.12.2018e

\begin{abstract}
The paper considers the problem of gated myocardial perfusion single photon emission computed tomography (SPECT) data processing. An approach to the quantitative analysis of gated myocardial perfusion SPECT data used in software developed in the SPbSU is presented. The article presents and formalizes the complete data processing workflow. All the main tasks of the data processing are considered. Mathematical representation of problem domain objects is presented. A detailed algorithm of the data processing is given. The algorithm is implemented as component of the data processing software suite.
\end{abstract}

\section{Key words}

Gated single photon emission computer tomography (gSPECT), myocardial perfusion imaging (MPI), software, image segmentation, functional images

\section{Introduction}

Development of mathematical models, algorithms and software for modern medical diagnostics is an important task. It helps improve the quality and information value of medical data and allows high-precision diagnostics in the early stages of the disease.

Methods of automatic data processing are intensively developed nowadays. Segmentation algorithms and data representation methods are being elaborated and improved [Xu et al., 2009; Germano et al., 2016; Kotina and Ploskikh, 2012; Kotina and Pasechnaya, 2014]. Methods of construction of parametric images in cardiology based on Fourier and wavelet analysis are evolving [Chen et al., 2005; Chen et al., 2011; Hsu et al., 2013; Babin and Kotina, 2014; Ostroumov et al., 2015; Nakajima et al., 2017].

\author{
Elena Kotina \\ Saint Petersburg State University \\ Russia \\ e.kotina@spbu.ru
}

Cardiological studies with the calculation of quantitative indicators are becoming increasingly common. There is a tendency to complete automation of processing and interpretation of radioisotope cardiac studies [Slomka et al., 2017]. For this purpose problems of the myocardium segmentation and quantitative analysis are considered [Germano and Slomka, 2017]. Research on this subject continues to be relevant [Slomka et al., 2016; Gomez et al., 2018].

The main software packages for the treatment of cardiac studies are developed at Cedars Sinai Medical Center (Los Angeles, USA) [Germano et al., 2007], Michigan University/INVIA, LLC (Ann arbor, USA) [Ficaro et al., 2007] and Emory University/Syntermed Inc. (Atlanta, USA) [Garcia et al., 2007].

The article considers the data processing in gated SPECT myocardial perfusion imaging. The data processing algorithm is developed, the main steps of the algorithm are described in detail.

The algorithm allows for fully automatic data processing, and also gives the expert the ability to adjust the intermediate results of the processing stages to improve the work in specific cases.

\section{Gated Myocardial Perfusion SPECT}

Electrocardiographically gated myocardial perfusion SPECT (gSPECT) is one of the most vital procedures in clinical cardiology. It allows to evaluate the left ventricular (LV) perfusion and function within a single study. Automation of the image processing and quantification has made this study highly reproducible and practical in the clinical setting. Comprehensive quantitative analysis of the data enhances the diagnostic and prognostic capability of myocardial perfusion imaging (MPI). GSPECT MPI has shown potentials for myocardial viability assessment and follow-up after therapy [Ostroumov et al., 2017]. MPI study can provide prognostic information for the prediction of major cardiovascular events and indications for revascularization 
therapy in patients with coronary artery disease.

The study is conducted using $\gamma$-camera with tomographic acquisition (single photon emission computer tomograph - SPECT) [Arlychev et al., 2009; Dorbala et al., 2018].

After gSPECT acquisition and reconstruction [Kotina et al., 2013a] we get a sequence of three-dimensional arrays containing information on the distribution of the radiopharmaceutical in the left ventricular myocardium at different intervals of the representative cardiac cycle [Kotina et al., 2013b].

To obtain prognostic information, it is necessary to perform mathematical processing of all data in order to obtain numerical values of significant parameters, graphs, polar maps and three-dimensional images of the left ventricle of the heart. Important diagnostic parameters that should be evaluated are the LV ejection fraction, end-diastolic and end-systolic volumes of the LV. To plot the changes in the volume of the LV during the cardiac cycle, the volumes for each interval of the representative cardiac cycle are determined. Various hemodynamic parameters are calculated according to the volume curve. The study is based on the fact that the distribution of the radiopharmaceutical in the myocardium reflects the distribution of perfusion in the heart muscle at different intervals of the cardiac cycle. Therefore, it is important to obtain a visual and quantitative assessment of this distribution. As a rule, it is visualized in the end-diastole and the end-systole, as well as an ungated version is considered. To assess LV function, it is necessary to obtain a quantitative and visual representation of systolic thickening, myocardial wall movement, as well as a numerical and visual assessment of LV dyssynchrony, which is of great importance for patients intent for resynchronization therapy [Ostroumov et al., 2011].

\section{Algorithm}

This section presents the algorithm of data processing of gSPECT MPI. Processing stages are mathematically formalized, the algorithm steps are described according to the sequence of their execution.

\subsection{Source Data}

The algorithm uses series of gated three-dimension cardiac images $V_{k}$ as input data, where $k=1 . . N$ is the gating interval index, $N=2 M-$ the even number of gating intervals which the duration of cardiac cycle $T$ is divided into. The images are considered as continuous functions of the arguments $(x, y, z)$ obtained from the original arrays by trilinear interpolation. Ungated image is defined as

$$
V(x, y, z)=\sum_{k=1}^{N} V_{k}(x, y, z) .
$$

The coordinate system for the trilinear interpolation is chosen so that the coordinate axes are oriented as follows: $x$ - septal-lateral, $y$ - anteroposterior, $z-$ apical-basal.

\subsection{Left Ventricle Segmentation}

The first step of the algorithm is left ventricle segmentation. The segmentation process is similar to one used in QPS[Germano et al., 1995].

The largest count value of the ungated image $\left(V_{\max }=\right.$ $\max (V(x, y, z)))$ is found. The image is then thresholded to $50 \% V_{\max }$ and binarized. Binary clusters (sets of 6-connected voxels) are determined.

Small clusters (less than $50 \mathrm{ml}$ ) are removed. If two or more clusters are obtained, the closest one to the image center is selected. If the selected cluster satisfies the aspect constraints (the difference in length and width is less than $15 \mathrm{~mm}$ ), then it is considered as myocardial. Otherwise the partition process is performed: the initial threshold is increased by $5 \%$ until the cluster is divided into several parts, then the dilation of the parts is performed until its union will be the original cluster, after which the selection process is repeated.

The segmentation algorithm is further enhanced by providing feature to manual specify three-dimensional region of interest (ROI) in the image, the values outside of which are ignored.

\subsection{Sampling Grid Definition}

The myocardial cluster obtained at the previous stage is skeletonized.

A set of skeletal points is approximated by an axisoriented ellipsoid. Its $x$ - and $y$-axes are considered equal (short axes). Its $z$-axis will be called long axis. The geographical grid $\left(\vec{r}_{i}, \vec{n}_{i}\right)$ defined on the ellipsoid surface is used for further calculations. The grid consists of 770 points: 24 non-polar latitude samples, 32 longitude samples, 2 poles.

Sampling grid vertex positions defined by formula

$$
\vec{r}_{i}=\left(\begin{array}{c}
R_{s} \cos \theta_{i} \cos \varphi_{i} \\
R_{s} \cos \theta_{i} \sin \varphi_{i} \\
R_{l} \sin \theta_{i}
\end{array}\right)
$$

and its normals

$$
\vec{n}_{i}=\left(\begin{array}{c}
R_{l} R_{s} \cos \theta_{i} \cos \varphi_{i} \\
R_{l} R_{s} \cos \theta_{i} \sin \varphi_{i} \\
R_{s}^{2} \sin \theta_{i}
\end{array}\right),
$$

where $R_{l}$ - long axis length, $R_{s}$ - short axis length, $\theta_{i}$ and $\varphi_{i}$ - latitude and longitude of $i$ th vertex defined as follows

$$
\theta_{i}=\left\{\begin{array}{rlrl}
-\frac{\pi}{2}+\frac{\pi}{25}(\lfloor(i-1) / 32\rfloor+1), & i=1 . .768 \\
-\frac{\pi}{2}, & i=769 \\
\frac{\pi}{2}, & i & =770
\end{array},\right.
$$




$$
\varphi_{i}=\left\{\begin{array}{cl}
\frac{\pi}{16}(i-1), i & =1 . .768 \\
0, & i=769 . .770
\end{array} .\right.
$$

The feature to manually adjust the elliptical coordinate system is provided to improve the algorithm behaviour in specific cases.

\subsection{Generation of Mid-myocardial Surfaces}

The mid-myocardial surface points $\vec{s}_{i}$ are defined as an offsets $D_{i}$ from the corresponding grid points $\vec{r}_{i}$ along the normals $\vec{n}_{i}$ :

$$
\vec{s}_{i}=\vec{r}_{i}+D_{i} \frac{\vec{n}_{i}}{\left\|\vec{n}_{i}\right\|}
$$

The mid-myocardial determination process starts with an approximation. Profiles along the ellipsoid normals are extracted at each point of the grid. Profiles length is constrained to $20 \mathrm{~mm}$ in both directions. The profile is then convolved with Lagrangian of Gaussian feature detector with a s.d. of $10 \mathrm{~mm}$. The displacement of the maxima $D_{i}^{*}$ is taken as the approximation.

The resulting surface is obtained by minimizing the weighted sum of the deviations of the surface points from the initial approximation and from the point's nearest neighbors. The points position is a solution of the system of linear equations

$$
\left(\left\|\mathfrak{N}_{i}\right\|+k_{v} V\left(\vec{s}_{i}^{*}\right)\right) D_{i}-\sum_{j \in \mathfrak{N}_{i}} D_{j}=k_{v} V\left(\vec{s}_{i}^{*}\right) D_{i}^{*},
$$

where $\mathfrak{N}_{i}$ is the set of indices of neighboring grid nodes for the $i$ th element ( 32 elements for the poles, 4 for the others), $k_{v}=\frac{1}{\max _{j} V\left(\vec{s}_{j}^{*}\right)}$ is the coefficient of influence of the perfusion values.

Gated mid-myocardial surfaces are constructed in a similar way with added temporal continuity constraint

$$
\begin{array}{r}
\left(\left\|\mathfrak{N}_{i}\right\|+k_{v} V_{k}\left(\vec{s}_{k, i}^{*}\right)+2 k_{t}\right) D_{k, i}-\sum_{j \in \mathfrak{N}_{i}} D_{k, j}- \\
-k_{t}\left(D_{k-1, i}+D_{k+1, i}\right)=k_{v} V_{k}\left(\vec{s}_{k, i}^{*}\right) D_{k, i}^{*}
\end{array}
$$

where $D_{k, i}$ is unknown displacement of $i$ th surface point along $i$ th grid normale over the $k$ th interval, $D_{k, i}^{*}$ is the initial approximation, $k_{v}=\frac{2}{\max _{k, \vec{x}} V_{k}(\vec{x})}$ is the coefficient of influence of the perfusion values, $k_{t}=1$ is the time continuity coefficient.

\subsection{Valve Plane Determination}

After mid-myocardial surfaces has been built the valve plane position can be determined. The total perfusion map is calculated

$$
P_{i}^{\text {sum }}=\sum_{k=1}^{N} V_{k}\left(\vec{s}_{k, i}\right)
$$

The initial threshold of $25 \%$ is set. A contiguous subthreshold region containing the basal pole is selected. Its border points are mapped to the mid-myocardial surface of the first interval and are approximated by a plane. The area of the surface basal to the valve plane is calculated. If the area is less than $10 \%$ of the total midmyocardial surface area, the threshold is raised and the process is repeated.

The resulting plane defines mask of surface points used to exclude surface points in subsequent gating intervals (fig. 1).

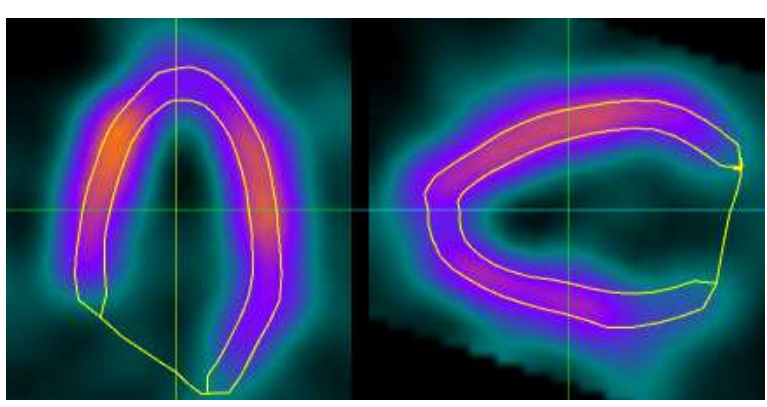

Figure 1. Myocardial surfaces constrained by valve plane

The feature to manually adjust the valve plane is provided to improve the algorithm behaviour in specific cases.

\subsection{Extraction of Epi- and Endocardial Surfaces}

The epi- $\left(\vec{s}^{+}\right)$and endocardia $1\left(\vec{s}^{-}\right)$surfaces are defined as an offsets $E^{+}$and $E^{-}$along the normals of the mid-myocardial surface as follows:

$$
\vec{s}_{i}^{+}=\vec{s}_{i}+E_{i}^{+} \frac{\vec{n}(s, i)}{\|\vec{n}(s, i)\|}
$$

$$
\begin{gathered}
\vec{s}_{i}=\vec{s}_{i}-E_{i}^{-} \frac{\vec{n}(s, i)}{\|\vec{n}(s, i)\|}, \\
\vec{n}(s, i)=\sum_{j=1}^{\left\|\mathfrak{N}_{i}\right\|} \frac{\left(\vec{s}_{n_{i, j}}-\vec{s}_{i}\right) \times\left(\vec{s}_{n_{i, j+1}}-\vec{s}_{i}\right)}{\left\|\left(\vec{s}_{n_{i, j}}-\vec{s}_{i}\right) \times\left(\vec{s}_{n_{i, j+1}}-\vec{s}_{i}\right)\right\|},
\end{gathered}
$$

where $n_{i, j} \in \mathfrak{N}_{i}$ is the index of $j$ th neighbour of $i$ th vertex, while $\vec{s}_{n_{i, j}}$ are ordered by $j$ clockwise around $\vec{s}_{i}$ with respect to the normal of $\vec{n}_{i}$.

An approximation $\left(E^{*+}\right.$ and $\left.E^{*-}\right)$ is made as follows. A profile along the direction normal to the surface extending $20 \mathrm{~mm}$ in both directions is extracted for each point of the myocardial surface. The value at the origin 
is taken as the maximum. The distance of $25 \%$ value drop (75\% threshold passing) is measured in both directions. If needed decrease in value is not registered, then the point is marked invalid. All invalid offsets are replaced by values that minimize the difference between offsets of neighboring nodes.

A scaling factor is introduced to eliminate the effects caused by different acquisition environments. It is chosen so that the average thickness of the myocardium for an ungated image will be $10 \mathrm{~mm}$ :

$$
\beta=\frac{10 \cdot 770}{\sum_{i}\left(E^{*+}-E^{*-}\right)} .
$$

The epi- and endocardial surfaces undergo further adjustment to accommodate perfusion changes in the wall motion calculation [Germano et al., 1997]. Surface offset values are calculated as

$$
\begin{gathered}
E_{k, i}=\beta \gamma_{k} L_{k, i} E_{k, i}^{*}, \\
L_{k, i}=\left(1-\alpha_{i}\right)+\alpha_{i} \delta_{k, i}, \\
\alpha_{i}=\frac{V_{1}\left(\vec{s}_{1, i}\right)}{\max _{j} V_{1}\left(\vec{s}_{1, j}\right)}, \\
\delta_{k, i}=\max \left(0.5, \min \left(\frac{V_{k}\left(\vec{s}_{k, i}\right)}{V_{1}\left(\vec{s}_{1, i}\right)}, 2\right)\right),
\end{gathered}
$$

where $\gamma_{k}$ is chosen in such a way that the volume of the myocardium remains constant. The sample of result surfaces is shown in fig. 2.
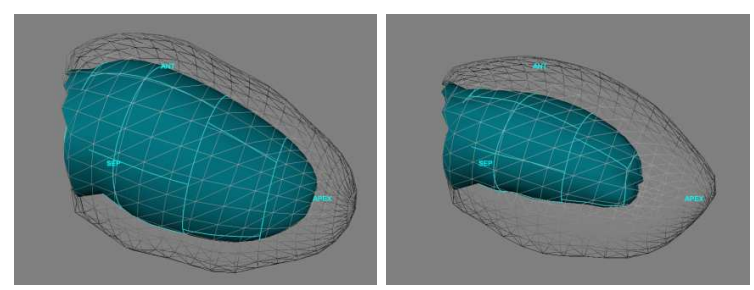

Figure 2. ED \& ES epi- and endocardial surfaces

\subsection{Volume Curve and Parameters Calculation}

For each interval we calculate volume of region constrained by endocardial surface and valve plane $v_{k}$.
Volume curve is defined via trigonometric interpolation of these values over cardiac cycle length:

$$
\begin{array}{r}
v(t)=\frac{a_{0}}{2}+\sum_{j=1}^{M}\left(a_{j} \cos \left(\frac{2 \pi}{T} t j\right)+\right. \\
\left.+b_{j} \sin \left(\frac{2 \pi}{T} t j\right)\right)+\frac{a_{M}}{2} \cos \left(\frac{2 \pi}{T} t M\right),
\end{array}
$$

where

$$
\begin{aligned}
a_{j} & =\frac{2}{N} \sum_{k=1}^{N} v_{k} \cos \left(\frac{2 \pi}{N} j(k-1)\right), \\
b_{j} & =\frac{2}{N} \sum_{k=1}^{N} v_{k} \sin \left(\frac{2 \pi}{N} j(k-1)\right) .
\end{aligned}
$$

Therefore the derivative is defined as follows

$$
\begin{array}{r}
v^{\prime}(t)=\sum_{j=1}^{M}\left(-\frac{2 \pi j a_{j}}{T} \sin \left(\frac{2 \pi}{T} t j\right)+\right. \\
\left.+\frac{2 \pi j b_{j}}{T} \cos \left(\frac{2 \pi}{T} t j\right)\right)-\frac{\pi M a_{M}}{T} \cos \left(\frac{2 \pi}{T} t M\right)
\end{array}
$$

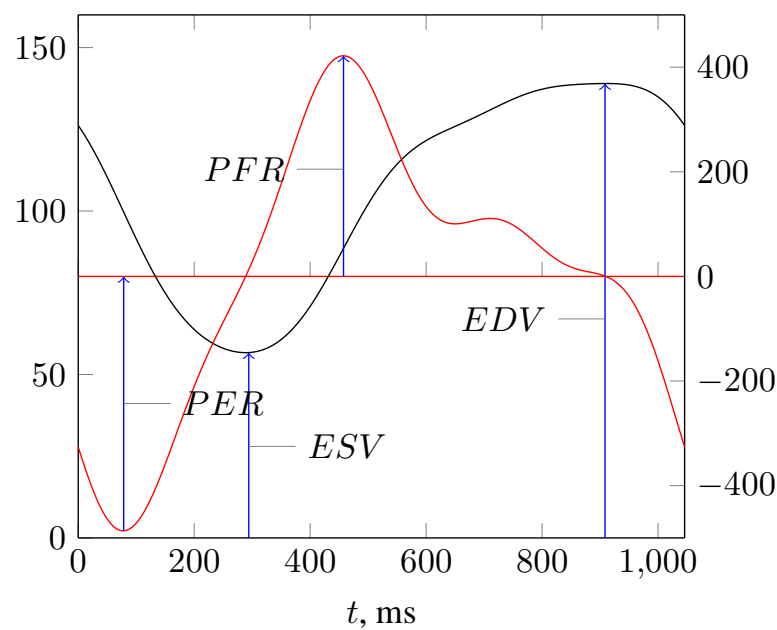

\section{— Volume $(\mathrm{ml}) \ldots$ Velocity $(\mathrm{ml} / \mathrm{s})$}

Figure 3. Hemodynamic curves and parameters

The end-diastole and end-systole times are defined as argument of volume curve maximum and minimum respectively:

$$
\begin{aligned}
& T_{E D}=\underset{x \in[0, T]}{\operatorname{argmax}} v(x), \\
& T_{E S}=\underset{x \in[0, T]}{\operatorname{argmin}} v(x) .
\end{aligned}
$$

Hemodynamic curves (fig. 3) are used to calculate main parameters by following formulae. 
End-diastolic volume:

$$
E D V=v\left(T_{E D}\right) .
$$

End-systolic volume:

$$
E S V=v\left(T_{E S}\right)
$$

Ejection fraction:

$$
E F=\left(1-\frac{E S V}{E D V}\right) \cdot 100 \%
$$

Stroke volume:

$$
S V=E D V-E S V
$$

Average ejection rate:

$$
A E R=\left\{\begin{array}{ll}
\frac{S V}{T_{E S}-T_{E D}} & \text { if } T_{E D}<T_{E S} \\
\frac{S V}{T-\left(T_{E S}-T_{E D}\right)} & \text { if } T_{E D} \geq T_{E S}
\end{array} .\right.
$$

Average filling rate:

$$
A F R=\left\{\begin{array}{ll}
\frac{S V}{T-\left(T_{E D}-T_{E S}\right)} & \text { if } T_{E D}<T_{E S} \\
\frac{S V}{T_{E D}-T_{E S}} & \text { if } T_{E D} \geq T_{E S}
\end{array} .\right.
$$

Peak ejection rate:

$$
P E R=\left\{\begin{array}{ll}
-\min _{x \in\left[T_{E D}, T_{E S}\right]} v^{\prime}(x) & \text { if } T_{E D}<T_{E S} \\
-\min _{x \in\left[0, T_{E S}\right] \cap\left[T_{E D}, T\right]} v^{\prime}(x) & \text { if } T_{E D} \geq T_{E S}
\end{array} .\right.
$$

Peak filling rate:

$$
P F R=\left\{\begin{array}{ll}
\max _{x \in\left[0, T_{E D}\right] \cap\left[T_{E S}, T\right]} v^{\prime}(x) & \text { if } T_{E D}<T_{E S} \\
\max _{x \in\left[T_{E S}, T_{E D}\right]} v^{\prime}(x) & \text { if } T_{E D} \geq T_{E S}
\end{array} .\right.
$$

\subsection{Parameters of Ventricular Perfusion and Function}

Ellipsoid grid allows us to extract standard number of data samples regardless of myocardial size. These samples represent state of distinct regions of myocardium and can be displayed two-dimensionally or three-dimensionally (fig. 4).

Two dimensional display (polar map) is based on polar azimuthal equidistant projection. About two thirds of data points adjacent to apex are used for display.
The rest are discarded as they usually located in basal area across the valve plane and do not represent myocardium. The sampling points then placed in unit circle as follows

$$
\left(\begin{array}{l}
x_{i} \\
y_{i}
\end{array}\right)=\frac{l\left(\theta_{i}\right)}{l\left(\frac{7 \pi}{50}\right)}\left(\begin{array}{c}
\cos \varphi_{i} \\
\sin \varphi_{i}
\end{array}\right)
$$

where $x_{i}^{2}+y_{i}^{2} \leq 1, l(\beta)-$ arc distance from latitude $\beta$ to apical pole

$$
l(\beta)=\int_{\beta}^{-\frac{\pi}{2}} \sqrt{1+\frac{R_{s}^{2}-R_{l}^{2}}{R_{l}^{2}} \sin ^{2} \theta} d \theta .
$$

The following regional parameters are used in data analysis.
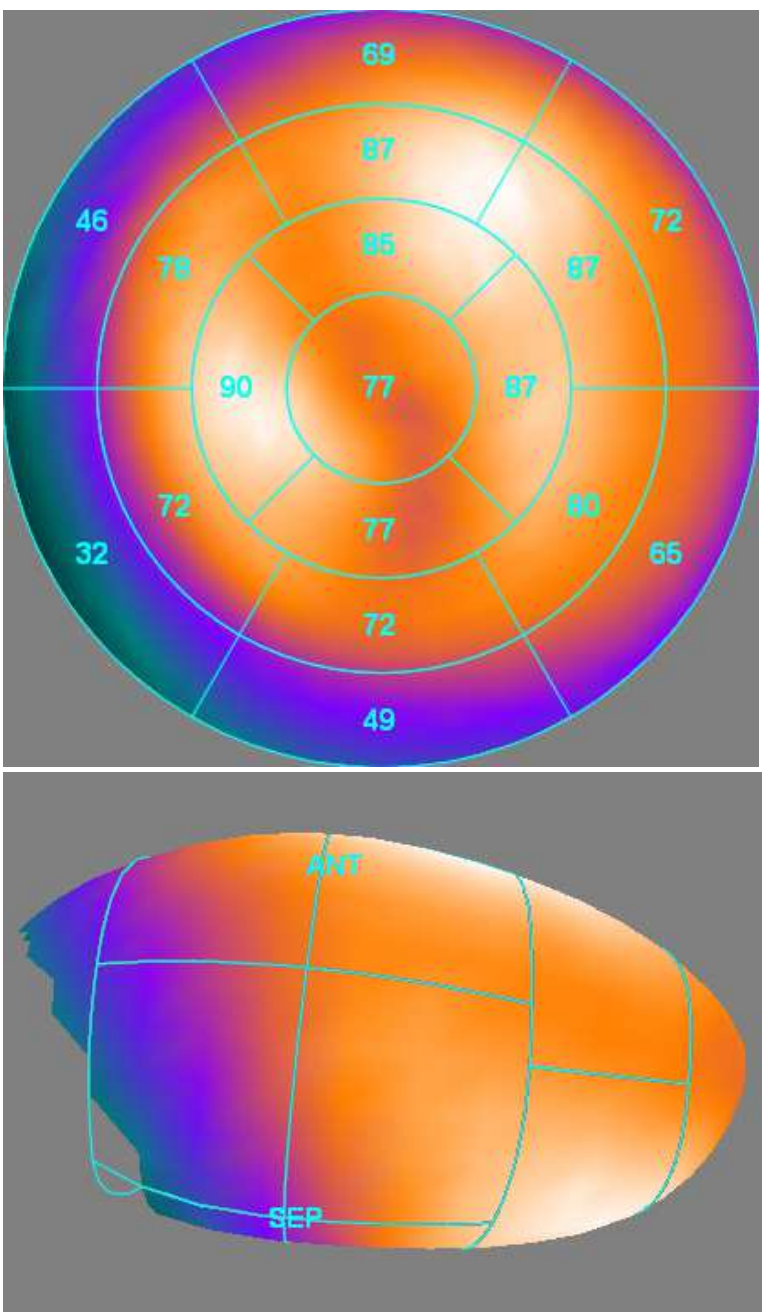

Figure 4. Perfusion map in 2D (top) and 3D (bottom) modes

Perfusion (fig. 4) is calculated as image value at cor- 


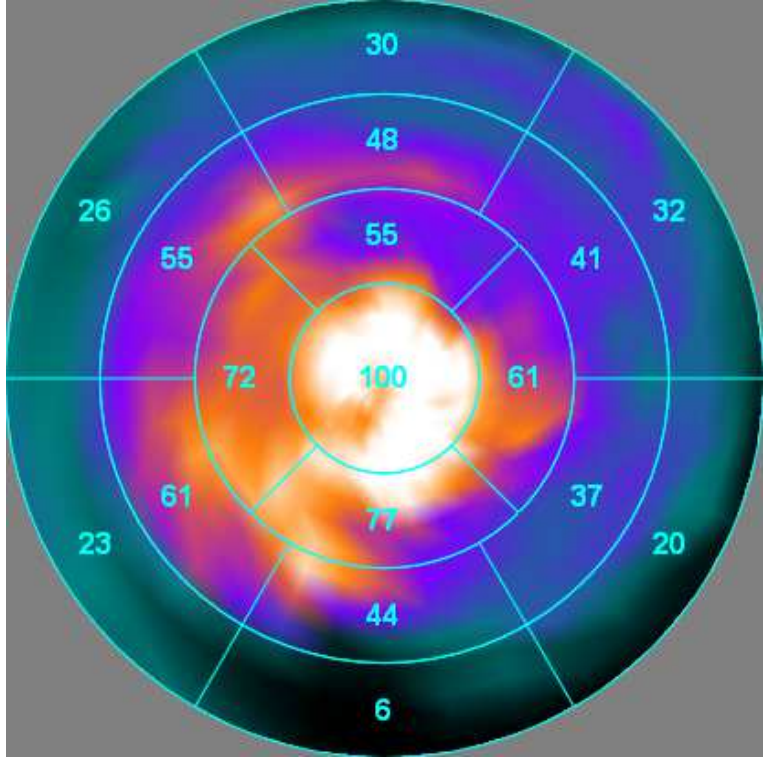

Figure 5. Thickening map

responding point of midmyocardial surface:

$$
P_{k, i}=V_{k}\left(\vec{s}_{k, i}\right) .
$$

Thickening (fig. 5) is computed as the variation in thickness between end-diastole and end-systole and is expressed as the percent increase from diastolic thickness.

$$
T H_{i}=\left(\frac{E_{E S, i}^{+}-E_{E S, i}^{-}}{E_{E D, i}^{+}-E_{E D, i}^{-}}-1\right) \cdot 100 \% .
$$

Wall motion (fig. 6) is measured as the distance between an endocardial surface at end-diastole and endocardial surface end-systole. The measurement is performed along normals to the average between enddiastole and end-systole mid-myocardial surfaces.

$$
\begin{gathered}
\vec{c}_{i}=\frac{1}{2}\left(\vec{s}_{E D, i}+\vec{s}_{E S, i}\right), \\
t_{k, i}=\min \left\{t \mid \vec{c}_{i}-t \vec{n}(c, i) \in s_{k}^{-}, t>0\right\}, \\
W M_{i}=t_{E D, i}-t_{E S, i} .
\end{gathered}
$$

LV mechanical dyssynchrony is measured by using phase analysis of gated myocardial perfusion SPECT. Phase map (fig. 7) is calculated as follows

$$
\phi_{i}=\operatorname{atan} 2\left(b_{i}, a_{i}\right),
$$

where

$$
\begin{aligned}
a_{i} & =\frac{2}{N} \sum_{k=1}^{N} P_{k, i} \cos \frac{2 \pi(k-1)}{N}, \\
b_{i} & =\frac{2}{N} \sum_{k=1}^{N} P_{k, i} \sin \frac{2 \pi(k-1)}{N} .
\end{aligned}
$$

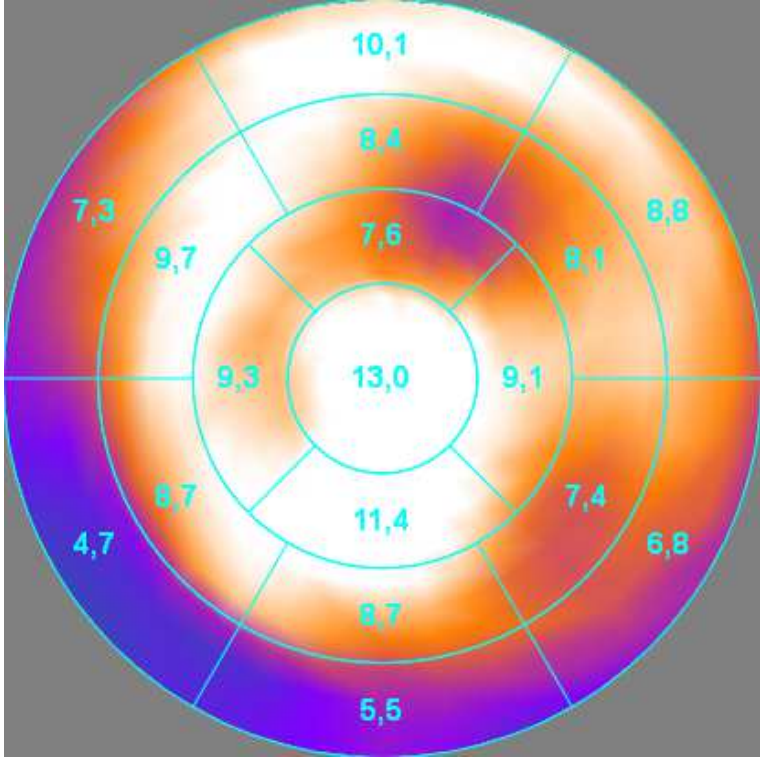

Figure 6. Wall motion map

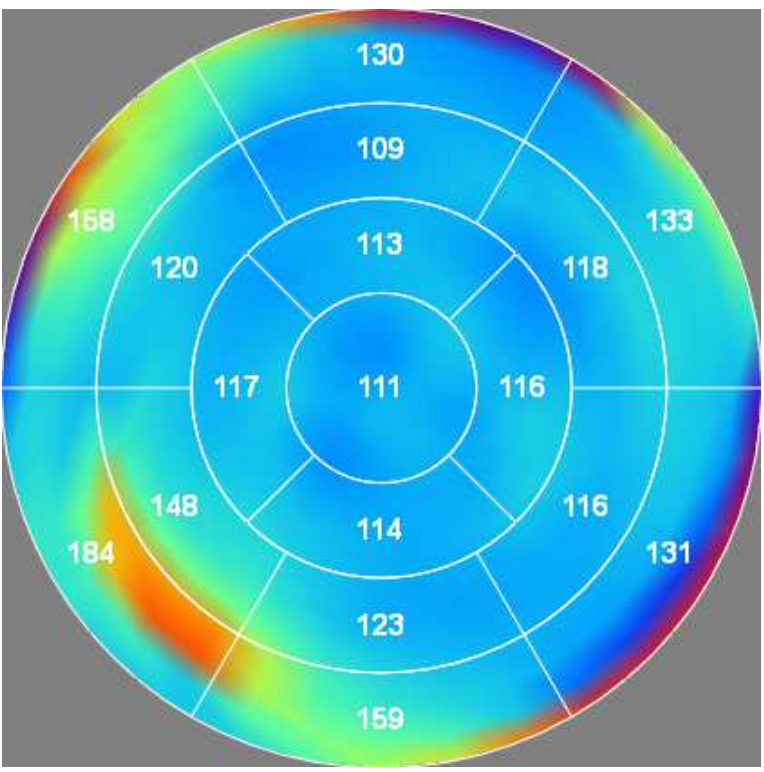

Figure 7. Phase map

\subsection{Phase and Magnitude Images}

The helpful feature of this algorithm is the construction of not only phase polar maps, but also phase and magnitude arrays (3D arrays), visualization of which is available at the first stage of segmentation for visual evaluation by an expert (or for making corrections at the stage of segmentation). Phase and magnitude images could be useful at the stage of segmentation, it is known that the atria and ventricles have opposite phase, and the magnitude value in the heart area significantly 


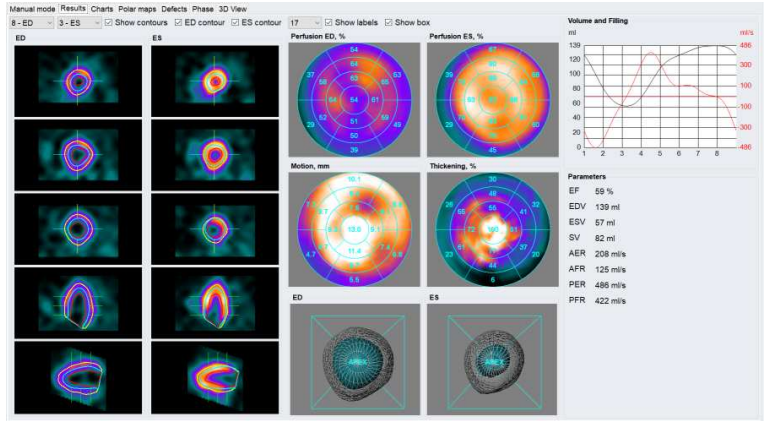

Figure 9. Software suite Indis

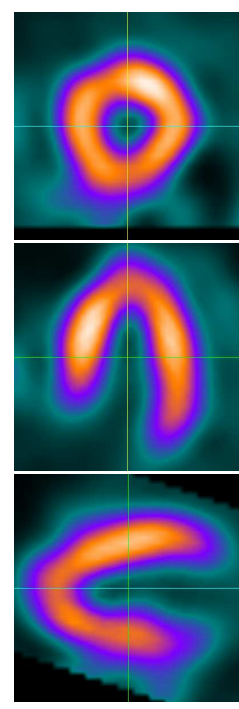

a)

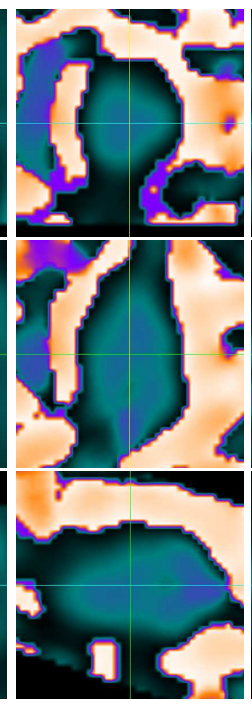

b)

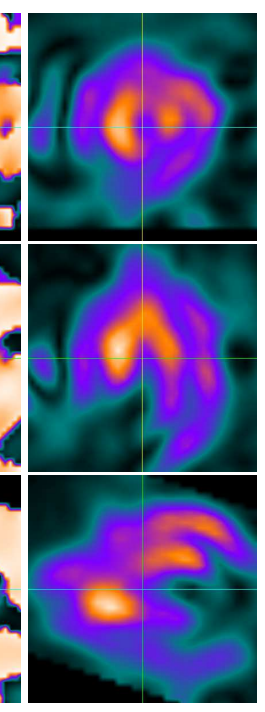

c)
Figure 8. Central slices of: a) ungated volume; b) phase volume c) magnitude volume

exceeds the magnitude from the surrounding heart areas. The slices of the phase and magnitude volumes are visualized (fig. 8). The volumes are constructed as follows

$$
V_{\phi}(x, y, z)=\operatorname{atan} 2\left(V_{b}(x, y, z), V_{a}(x, y, z)\right)
$$

$$
V_{M A G}=\sqrt{V_{a}^{2}(x, y, z)+V_{b}^{2}(x, y, z)},
$$

where

$$
\begin{aligned}
& V_{a}(x, y, z)=\frac{2}{N} \sum_{k=1}^{N} V_{k}(x, y, z) \cos \frac{2 \pi(k-1)}{N}, \\
& V_{b}(x, y, z)=\frac{2}{N} \sum_{k=1}^{N} V_{k}(x, y, z) \sin \frac{2 \pi(k-1)}{N} .
\end{aligned}
$$

\section{Conclusion}

An automatic algorithm to measure quantitatively myocardial perfusion SPECT is developed. The quantification algorithm consists of six main steps: the left ventricular myocardium segmentation, sampling grid definition, the left ventricle's surfaces extraction, valve plane determination, volume curve and hemodynamic parameters calculation, parameters of left ventricular perfusion and function evaluation and visualization.

The algorithm is implemented in Indis data processing suite (fig. 9), tested on clinical patient studies acquired with 16- and 8-interval gating and showed reproducible results.

\section{References}

Arlychev, M. A., Novikov, V. L., Sidorov, A. V., Fialkovskii, A. M., Kotina, E. D., Ovsyannikov, D. A., and Ploskikh, V. A. (2009). EFATOM two-detector one-photon emission gamma tomograph. Technical Physics, 54(10):1539-1547.

Babin, A. V. and Kotina, E. D. (2014). Mathematical data processing of gated SPECT myocardial perfusion imaging with using wavelet analysis. 2014 International Conference on Computer Technologies in Physical and Engineering Applications, ICCTPEA 2014 - Proceedings, pages 19-20.

Chen, J., Garcia, E. V., Folks, R. D., Cooke, C. D., Faber, T. L., Tauxe, E. L., and Iskandrian, A. E. (2005). Onset of left ventricular mechanical contraction as determined by phase analysis of ECG-gated myocardial perfusion SPECT imaging: development of a diagnostic tool for assessment of cardiac mechanical dyssynchrony. J Nucl Cardiol, 12(6):687695.

Chen, J., Kalogeropoulos, A. P., Verdes, L., Butler, J., and Garcia, E. V. (2011). Left-ventricular systolic and diastolic dyssynchrony as assessed by multiharmonic phase analysis of gated SPECT myocardial perfusion imaging in patients with end-stage renal disease and normal LVEF. J Nucl Cardiol, 18(2):299-308.

Dorbala, S., Ananthasubramaniam, K., Armstrong, I. S., Chareonthaitawee, P., DePuey, E. G., Einstein, A. J., Gropler, R. J., Holly, T. A., Mahmarian, J. J., Park, M. A., Polk, D. M., Russell, R., Slomka, P. J., Thompson, R. C., and Wells, R. G. (2018). Single Photon Emission Computed Tomography (SPECT) Myocardial Perfusion Imaging Guidelines: Instrumentation, Acquisition, Processing, and Interpretation. J Nucl Cardiol, 25(5):1784-1846.

Ficaro, E. P., Lee, B. C., Kritzman, J. N., and Corbett, J. R. (2007). Corridor4DM: the Michigan method for quantitative nuclear cardiology. J Nucl Cardiol, 14(4):455-465.

Garcia, E. V., Faber, T. L., Cooke, C. D., Folks, R. D., Chen, J., and Santana, C. (2007). The increasing role of quantification in clinical nuclear cardiology: the Emory approach. J Nucl Cardiol, 14(4):420-432. 
Germano, G., Erel, J., Lewin, H., Kavanagh, P. B., and Berman, D. S. (1997). Automatic quantitation of regional myocardial wall motion and thickening from gated technetium-99m sestamibi myocardial perfusion single-photon emission computed tomography. J. Am. Coll. Cardiol., 30(5):1360-1367.

Germano, G., Kavanagh, P. B., Fish, M. B., Lemley, M. H., Xu, Y., Berman, D. S., and Slomka, P. J. (2016). Same-Patient Processing" for multiple cardiac SPECT studies. 1. Improving LV segmentation accuracy. J Nucl Cardiol, 23(6):1435-1441.

Germano, G., Kavanagh, P. B., Slomka, P. J., Van Kriekinge, S. D., Pollard, G., and Berman, D. S. (2007). Quantitation in gated perfusion SPECT imaging: the Cedars-Sinai approach. $J$ Nucl Cardiol, 14(4):433-454.

Germano, G., Kiat, H., Kavanagh, P. B., Moriel, M., Mazzanti, M., Su, H. T., Van Train, K. F., and Berman, D. S. (1995). Automatic quantification of ejection fraction from gated myocardial perfusion SPECT. J. Nucl. Med., 36(11):2138-2147.

Germano, G. and Slomka, P. J. (2017). Assessing LV remodeling in nuclear cardiology. J Nucl Cardiol.

Gomez, J., Doukky, R., Germano, G., and Slomka, P. (2018). New Trends in Quantitative Nuclear Cardiology Methods. Curr Cardiovasc Imaging Rep, 11(1).

Hsu, T. H., Huang, W. S., Chen, C. C., Hung, G. U., Chen, T. C., Kao, C. H., and Chen, J. (2013). Left ventricular systolic and diastolic dyssynchrony assessed by phase analysis of gated SPECT myocardial perfusion imaging: a comparison with speckle tracking echocardiography. Ann Nucl Med, 27(8):764771.

Kotina, E. and Pasechnaya, G. A. (2014). Optical flowbased approach for the contour detection in radionuclide images processing. Cybernetics and physics, 3(2):62-65.

Kotina, E. and Ploskikh, V. A. (2012). Data processing and quantitation in nuclear medicine. RuPAC 2012 Contributions to the Proceedings - 23rd Russian Particle Accelerator Conference, pages 526-528.

Kotina, E. D., Latypov, V. N., and Ploskikh, V. A. (2013a). Universal system for tomographic recon- struction on gpus. Problems of Atomic Science and Technology, 88(6):175-178.

Kotina, E. D., Ploskikh, V. A., and Babin, A. B. (2013b). Radionuclide methods application in cardiac studies. Problems of Atomic Science and Technology, 88(6):179-182.

Nakajima, K., Okuda, K., Matsuo, S., and Slomka, P. (2017). Making the invisible visible: Phase dyssynchrony has potential as a new prognostic marker. $J$ Nucl Cardiol.

Ostroumov, E. N., Kotina, E. D., Ploskikh, V. A., Babin, A. V., Ivanov, A. S., Rodionov, A. V., and Mas, O. M. (2015). Visualization of acute myocardial infarction of right ventricle free wall after cardiac surgery by gated SPECT with phase images. European Journal of Nuclear Medicine and Nuclear Imaging, 42(1):S509.

Ostroumov, E. N., Kotina, E. D., Slobodyanik, V. V., Tonkoshkurova, V. V., Tunyaeva, I., Ermolenko, A. E., and Shumakov, D. V. (2011). ECGsynchronized myocardial perfusion tomoscintigraphy and cardiac resynchronization therapy. Russian Journal of Cardiology, 89(3):58-65.

Ostroumov, E. N., Migunova, E. V., Kotina, E. D., Sinyakova, O. G., Gazaryan, G. A., Ryabinin, V. A., and Kudryashova, N. E. (2017). What changes in perfusion and myocardial function after late revascularization of acute myocardial infarction? Sklifosovsky Journal Emergency Medical Care, 6(2):118-1123.

Slomka, P., Hung, G. U., Germano, G., and Berman, D. S. (2016). Novel SPECT Technologies and Approaches in Cardiac Imaging. Cardiovasc Innov Appl, 2(1):31-46.

Slomka, P. J., Dey, D., Sitek, A., Motwani, M., Berman, D. S., and Germano, G. (2017). Cardiac imaging: working towards fully-automated machine analysis \& interpretation. Expert Rev Med Devices, 14(3):197-212.

Xu, Y., Kavanagh, P., Fish, M., Gerlach, J., Ramesh, A., Lemley, M., Hayes, S., Berman, D. S., Germano, G., and Slomka, P. J. (2009). Automated quality control for segmentation of myocardial perfusion SPECT. J. Nucl. Med., 50(9):1418-1426. 